\title{
HÖLDER ESTIMATES FOR GREEN'S FUNCTIONS ON CONVEX POLYHEDRAL DOMAINS AND THEIR APPLICATIONS TO FINITE ELEMENT METHODS.
}

\author{
J. GUZMÁN, D. LEYKEKHMAN, J. ROSSMANN, AND A.H. SCHATZ
}

\begin{abstract}
A model second-order elliptic equation on a general convex polyhedral domain in three dimensions is considered. The aim of this paper is twofold: First sharp Hölder estimates for the corresponding Green's function are obtained. As an applications of these estimates to finite element methods, we show the best approximation property of the error in $W_{\infty}^{1}$. In contrast to previously known results, $W_{p}^{2}$ regularity for $p>3$, which does not hold for general convex polyhedral domains, is not required. Furthermore, the new Green's function estimates allow us to obtain localized error estimates at a point.
\end{abstract}

\section{INTRODUCTION}

In this paper we consider the model second-order elliptic problem

$$
\begin{aligned}
-\Delta u=f, & \text { in } \Omega, \\
u=0, & \text { on } \partial \Omega,
\end{aligned}
$$

where $\Omega$ is a convex polyhedral domain in three dimensions and $f$ is a smooth function. It is well known that for the above problem there exists a unique solution in $H^{2}(\Omega)$ (cf. [12]).

Let $S_{h}$ be a finite dimensional subspace of $H_{0}^{1}(\Omega)$ composed of piecewise polynomials of degree $k$ on a quasi-uniform mesh of size $h$ and $u_{h} \in S_{h}$ be the finite element approximation to $u$,

$$
\left(\nabla u_{h}, \nabla \chi\right)_{\Omega}=(\nabla u, \nabla \chi)_{\Omega}, \quad \forall \chi \in S_{h},
$$

where $(\nabla u, \nabla v)_{S}=\int_{S} \nabla u \cdot \nabla v$.

Our motivation is to establish the following best approximation property

$$
\left\|\nabla\left(u-u_{h}\right)\right\|_{L^{\infty}(\Omega)} \leq C \inf _{\chi \in S_{h}}\|\nabla(u-\chi)\|_{L^{\infty}(\Omega)},
$$

with constant $C$ independent of $h$. Such a result has many applications. For example, 1.2 is needed in order to establish the numerically observed $L^{2}$ error estimate for bi-harmonic problems (cf. [37]). By taking $\chi=0$ in 1.2 , we obtain the following stability result

$$
\left\|\nabla u_{h}\right\|_{L^{\infty}(\Omega)} \leq C\|\nabla u\|_{L^{\infty}(\Omega)},
$$

Date: September 10, 2007.

1991 Mathematics Subject Classification. 65N30,65N15.

Key words and phrases. Green's function, Hölder estimates, finite element, optimal error estimates. 
which is essential, for example, in analyzing the finite element solution of nonlinear problems (cf. 6, 7, 9, 11, 29]).

Many important contributions have been made in order to establish 1.2 with various assumptions on the finite element spaces and geometry of $\Omega$. Here we highlight some of the contributions. The first results valid for general quasi-uniform meshes were obtained by Natterer 23 and Scott 38. Natterer treated piecewise linear approximation on convex polygonal domains and Scott treated the problem with Neumann boundary conditions in two dimensions. Rannacher [27] and Nitsche 24, 25] considered the problem for arbitrary order approximations and on a smooth domain $\Omega$. Schatz [30] considered non-convex polygonal domains and used a discrete maximum principle in his proof. All the above results were sub-optimal (contained a logarithmic factor) for piecewise linear elements. In 1982, Rannacher and Scott in 28] proved optimal error estimates for convex polygonal domains and smooth domains. In the book by Brenner and Scott [3], these results were extended to three dimensional polyhedral domains with certain restrictions on the geometry.

The main analytical tool used in $3,24,25,27,28]$ was the fact that it is possible to obtain $L^{\infty}$ bounds from certain weighted $L^{2}$ estimates. However, the drawback of the above technique is that it does not give optimal error estimates for general polyhedral domains in three dimensions. For example, the estimates contained in [3] require $W_{p}^{2}$ regularity, where $p$ must be strictly greater than the space dimension. Although such regularity holds for general convex polygonal domains, in three dimensions such result puts strong restrictions on the geometry of polyhedral domains. More specifically, it requires that inner dihedral angles be smaller than $3 \pi / 4$ (cf. Theorem 7.1 in [21]). This restriction is unnatural since it is known that for any convex polyhedral domain $\Omega$ the solution $u$ to $(1.1)$ is in $W_{\infty}^{1}(\Omega)$. Actually, the gradient of the solution is Hölder continuous, where the modulus of the continuity depends on the geometry of the domain. Using imbedding theorems, this result can be deduced from the regularity results in weighted Sobolev spaces (cf. [19]). For the Stokes system such $C^{1+\sigma}$ result is established for the velocity field in [22].

A different technique was developed by Schatz and Wahlbin (cf. [31, 34, 35, 36]). In those papers instead of using global weighted $L^{2}$ error estimates, they used local $L^{2}$ error estimates (cf. 26]), along with dyadic decompositions of $\Omega$. The technique is independent of dimension, but relies on sharp pointwise bounds for high-order derivatives of the Green's function. These types of the Green's function estimates are known for smooth domains [18, but do not hold for general convex polyhedral domains (cf. [12, 15]).

Carefully examining the arguments of Schatz and Wahlbin, one can notice that in order to establish 1.2, it is sufficient to have certain Hölder type estimates for the first order derivatives and the second order mixed derivatives of the Green's function. More precisely, one would require for some $\sigma>0$, which may depend on the geometry of the domain $\Omega$,

$$
\begin{aligned}
\frac{\left|\partial_{x_{i}} G(x, \xi)-\partial_{y_{i}} G(y, \xi)\right|}{|x-y|^{\sigma}} & \leq C\left(|x-\xi|^{-2-\sigma}+|y-\xi|^{-2-\sigma}\right), \\
\frac{\left|\partial_{x_{i}} \partial_{\xi_{j}} G(x, \xi)-\partial_{y_{i}} \partial_{\xi_{j}} G(y, \xi)\right|}{|x-y|^{\sigma}} & \leq C\left(|x-\xi|^{-3-\sigma}+|y-\xi|^{-3-\sigma}\right),
\end{aligned}
$$

for $i, j=1,2,3$. 
Therefore, the main contribution of this papers is twofold. First we establish Hölder estimates for the Green's function (1.4), then using these estimates we prove the best approximation property $(1.2)$ for the finite element method for a general convex polyhedral domain.

Now we comment on 1.4. If $\Omega \subset \mathbb{R}^{3}$ has a smooth boundary, the following estimate holds (cf. [18])

$$
\left|D_{x}^{\alpha} D_{\xi}^{\beta} G(x, \xi)\right| \leq \frac{C}{|x-\xi|^{1+|\alpha|+|\beta|}} .
$$

If $\partial \Omega$ is not smooth, then in general the right-hand side of the above estimate will be a function of the distance of $x$ and $\xi$ to the singularities of $\partial \Omega$ (cf. [19]). However, in the case $\Omega$ is convex, but not necessarily a polyhedral domain, the above estimates are known to hold for $|\alpha| \leq 1$ and $|\beta| \leq 1$ (cf. [12, 15]). The counter-example given by Fromm [12] indicates that (1.5) with restrictions $|\alpha| \leq 1$ and $|\beta| \leq 1$ is sharp, in the sense that the estimates (1.4) do not hold for general convex domains. However, in the case of $C^{1+\sigma}$ boundaries, estimates of the type (1.4) were proved in [15]. In this paper we show that (1.4) also holds for convex polyhedral domains $\Omega$, where $\sigma>0$ depends on the geometry of $\Omega$. For example, if $\Omega$ is a cube, then the estimates (1.4) are valid for arbitrary $0<\sigma<1$.

Finally, we would like to present an improvement to $(1.2)$, weighted pointwise error estimates. Until 1998, all the pointwise error estimates were global, in the sense that the error at a point $z \in \Omega$ depended equally on the smoothness of $u$ on the whole domain $\Omega$. However, in [31, for smooth domains Schatz proved weighted pointwise error estimates that showed that the error at a point $z \in \Omega$ depends strongly on the behavior of $u$ in the vicinity of $z$ and rather weakly on the behavior of $u$ far from $z$. Here we prove a similar weighted result for convex polyhedral domains. More specifically, we show that for an arbitrary point $z \in \Omega$,

$$
\left|\nabla\left(u-u_{h}\right)(z)\right| \leq C \inf _{\chi \in S_{h}}\left\|\omega^{s} \nabla(u-\chi)\right\|_{L^{\infty}(\Omega)},
$$

where the weight $\omega(y)=\omega_{z, h}(y)=\frac{h}{h+|z-y|}$ and the power $s$ satisfies $0 \leq s<\sigma$. This is in contrast to smooth domains, where the power $s$ can be taken as high as the order of the approximating polynomials (cf. [31]). Notice that if we choose $s=0$ then the estimate reduces to 1.2 . However, for $s>0$ we have an improvement over (1.2). Finally we would like to point that the error estimate $(1.6)$ is the first $a$ priori weighted error estimate proved for non-smooth domains which holds up to the boundary.

Such weighted results proved to be very fruitful and sprang many interesting applications, for example, asymptotic error expansion inequalities (cf. [1, 31, 32]), superconvergence (cf. [33]), a posteriori averaging technique (cf. [4, 16]), a posteriori residual type estimators (cf. [6]), localized pointwise error estimates for quasilinear problems (cf. [8]), and Richardson Extrapolation (cf. [2]).

The rest of the paper is organized as follows. The next two sections are devoted to the proof of Green's function estimates (1.4). The proof is given for more general polyhedral type domains. In Section 4 we concentrate on the application of the Green's function estimates to finite element method. Thus, in Subsection 4.1 we state the basic assumptions on the mesh and the finite element spaces and in Subsection 4.2 we give a proof of the best approximation property 1.2 . In Subsection 
(4.3) we state the localized pointwise estimate (1.6). Finally, in the last section we comment on possible generalizations and extension to more complicated systems.

\section{Maximum modulus estimates for the Green's function in POLYHEDRAL DOMAINS}

2.1. The domain. Although for our finite element error estimates we will only consider convex polyhedral domains (flat faces and straight edges) we will prove (1.4) for more general polyhedral type domains (curved faces and edges).

In the proof of the Green's function estimates we assume that $\Omega$ is a bounded domain of polyhedral type in $\mathbb{R}^{3}$. This means that

(i) the boundary $\partial \Omega$ consists of smooth (of class $C^{\infty}$ ) open two-dimensional manifolds $\Gamma_{j}$ (the faces of $\Omega$ ), $j=1, \ldots, N$, smooth curves $M_{k}$ (the edges), $k=1, \ldots, N^{\prime}$, and corners $x^{(1)}, \ldots, x^{(d)}$,

(ii) for every $\xi \in M_{k}$ there exist a neighborhood $\mathcal{U}_{\xi}$ and a diffeomorphism (a $C^{\infty}$ mapping) $\kappa_{\xi}$ which maps $\Omega \cap \mathcal{U}_{\xi}$ onto $\mathcal{D}_{\xi} \cap B_{1}$, where $\mathcal{D}_{\xi}$ is a dihedron of the form

$$
\left\{x=\left(x_{1}, x_{2}, x_{3}\right) \in \mathbb{R}^{3}: 0<r<\infty, 0<\varphi<\theta_{\xi}, x_{3} \in \mathbb{R}\right\}
$$

(here $r, \varphi$ are the polar coordinates in the $\left(x_{1}, x_{2}\right)$-plane) and $B_{1}$ is the unit ball,

(iii) for every corner $x^{(j)}$ there exist a neighborhood $\mathcal{U}_{j}$ and a diffeomorphism $\kappa_{j}$ mapping $\Omega \cap \mathcal{U}_{j}$ onto $\mathcal{K}_{j} \cap B_{1}$, where

$$
\mathcal{K}_{j}=\left\{x \in \mathbb{R}^{3}: x /|x| \in X_{j}\right\}
$$

is a cone with vertex at the origin.

The domains $X_{j}$ in (iii) are subdomains of the unit sphere $S^{2}$ of polygonal type.

2.2. Notation. Let $\xi \in M_{k}$ be an edge point, and let $\Gamma_{k_{+}}, \Gamma_{k_{-}}$be the faces of $\Omega$ adjacent to $\xi$. Then by $\mathcal{D}_{\xi}$, we denote the dihedron which is bounded by the half-planes $\Gamma_{k_{ \pm}}^{\circ}$ tangential to $\Gamma_{k_{ \pm}}$at $\xi$. The angle between $\Gamma_{k_{+}}^{\circ}$ and $\Gamma_{k_{-}}^{\circ}$ is denoted by $\theta(\xi)$. We set

$$
\theta_{k}=\sup _{\xi \in M_{k}} \theta(\xi), \quad \mu_{k}=\pi / \theta_{k}, \quad \text { and } \quad \mu(x)=\pi / \theta_{k(x)},
$$

where $M_{k(x)}$ is the nearest edge to $x$ (more precisely, $k(x)$ is the smallest $k$ such that $\left.r_{k}(x)=r(x)\right)$. Let $x^{(j)}$ be a vertex of $\Omega$, and let $I_{j}$ be the set of all indices $k$ such that $x^{(j)}$ is an end-point of the edge $M_{k}$. By our assumptions on $\Omega$, there exist a neighborhood $\mathcal{U}_{j}$ of $x^{(j)}$ and a diffeomorphism $\kappa_{j}$ mapping $\Omega \cap \mathcal{U}_{j}$ onto a subset of the cone (2.1). Without loss of generality, we may assume that the Jacobian matrix $\kappa^{\prime}(x)$ coincides with the identity matrix at $x^{(j)}$. We denote by $\lambda_{j}$ the smallest eigenvalue of the Laplace-Beltrami operator $-\delta$ on the domain $X_{j}$ (with Dirichlet boundary conditions). Furthermore, let

$$
\Lambda_{j}=-\frac{1}{2}+\sqrt{\frac{1}{4}+\lambda_{j}}
$$

for $j=1, \ldots, d$. This means that $\lambda_{j}=\Lambda_{j}\left(\Lambda_{j}+1\right)$. 
2.3. Point estimates of the Green's function. Let $G(x, \xi)$ be the Green's function of the Dirichlet problem to the Laplace equation, i.e. $G(x, \xi)$ is the solution of the problem

$$
\begin{aligned}
-\Delta_{x} G(x, \xi) & =\delta(x-\xi) \text { for } x, \xi \in \Omega, \\
G(x, \xi) & =0 \text { for } x \in \partial \Omega, \xi \in \Omega .
\end{aligned}
$$

In the following, let $\mathcal{V}_{j}$ be a neighborhood of the vertex $x^{(j)}$ which has a positive distance to the edges $M_{k}, k \notin I_{j}$. Furthermore, let $\rho_{j}(x)$ be the distance of the point $x$ from the vertex $x^{(j)}, r_{k}(x)$ the distance from the edge $M_{k}$ and $r(x)=\min _{k} r_{k}(x)$ the distance from the set of all edge points.

The following estimates of $G(x, \xi)$ were proved in [19] (cf. also [20]).

(1) If $x, \xi \in \Omega \cap \mathcal{V}_{j}$, and $\rho_{j}(\xi)<2 \rho_{j}(x) / 3$, then

$$
\begin{aligned}
\left|D_{x}^{\alpha} D_{\xi}^{\gamma} G(x, \xi)\right| \leq & c_{\alpha, \gamma} \rho_{j}(x)^{-1-\Lambda_{j}-|\alpha|+\varepsilon} \rho_{j}(\xi)^{\Lambda_{j}-|\gamma|-\varepsilon} \\
& \times \prod_{k \in I_{j}}\left(\frac{r_{k}(x)}{\rho_{j}(x)}\right)^{\mu_{k}-|\alpha|-\varepsilon} \prod_{k \in I_{j}}\left(\frac{r_{k}(\xi)}{\rho_{j}(\xi)}\right)^{\mu_{k}-|\gamma|-\varepsilon},
\end{aligned}
$$

where $\varepsilon$ is an arbitrarily small positive number. The constant $c_{\alpha, \gamma}$ is independent of $x$ and $\xi$.

(2) If $x, \xi \in \Omega \cap \mathcal{V}_{j}$, and $\rho_{j}(\xi)>3 \rho_{j}(x) / 2$, then

$$
\begin{aligned}
\left|D_{x}^{\alpha} D_{\xi}^{\gamma} G(x, \xi)\right| \leq & c_{\alpha, \gamma} \rho_{j}(x)^{\Lambda_{j}-|\alpha|-\varepsilon} \rho_{j}(\xi)^{-1-\Lambda_{j}-|\gamma|+\varepsilon} \\
& \times \prod_{k \in I_{j}}\left(\frac{r_{k}(x)}{\rho_{j}(x)}\right)^{\mu_{k}-|\alpha|-\varepsilon} \prod_{k \in I_{j}}\left(\frac{r_{k}(\xi)}{\rho_{j}(\xi)}\right)^{\mu_{k}-|\gamma|-\varepsilon} .
\end{aligned}
$$

(3) If $x, \xi \in \Omega \cap \mathcal{V}_{j}, \rho_{j}(x) / 3<\rho_{j}(\xi)<3 \rho_{j}(x)$ and $|x-\xi|>\frac{2}{3} \min (r(x), r(\xi))$, then

$$
\left|D_{x}^{\alpha} D_{\xi}^{\gamma} G(x, \xi)\right| \leq c_{\alpha, \gamma}|x-\xi|^{-1-|\alpha|-|\gamma|}\left(\frac{r(x)}{|x-\xi|}\right)^{\mu(x)-|\alpha|-\varepsilon}\left(\frac{r(\xi)}{|x-\xi|}\right)^{\mu(\xi)-|\gamma|-\varepsilon} .
$$

(4) If $x, \xi \in \Omega \cap \mathcal{V}_{j}, \rho_{j}(x) / 3<\rho_{j}(\xi)<3 \rho_{j}(x)$ and $|x-\xi|<\min (r(x), r(\xi))$, then

$$
\left|D_{x}^{\alpha} D_{\xi}^{\gamma} G(x, \xi)\right| \leq c_{\alpha, \gamma}|x-\xi|^{-1-|\alpha|-|\gamma|} .
$$

(5) If $x$ and $\xi$ lie in neighborhoods $\mathcal{V}_{i}$ and $\mathcal{V}_{j}$ of different vertices $x^{(i)}$ and $x^{(j)}$ and $|x-\xi|>\delta$, where $\delta$ is a fixed positive number, then

$$
\begin{aligned}
\left|D_{x}^{\alpha} D_{\xi}^{\gamma} G(x, \xi)\right| \leq & c_{\alpha, \gamma} \rho_{i}(x)^{\Lambda_{i}-|\alpha|-\varepsilon} \rho_{j}(\xi)^{\Lambda_{j}-|\gamma|-\varepsilon} \\
& \times \prod_{k \in I_{i}}\left(\frac{r_{k}(x)}{\rho_{i}(x)}\right)^{\mu_{k}-|\alpha|-\varepsilon} \prod_{k \in I_{j}}\left(\frac{r_{k}(\xi)}{\rho_{j}(\xi)}\right)^{\mu_{k}-|\gamma|-\varepsilon} .
\end{aligned}
$$

From this point on we will assume that the domain $\Omega$ satisfies the following conditions.

$\mathbb{C} 1: X_{j}$ is a proper subset of the half-sphere for all $j$,

$\mathbb{C} 2: \theta_{k}<\pi$ for all $k$.

Condition $\mathbb{C} 2$ implies that $\mu_{k}>1$ for all $k$. Condition $\mathbb{C} 1$ will allow us to use the following result which can be found for example in [17.

Proposition 2.1. If $X_{j}$ is a proper subset of the half-sphere then $\Lambda_{j}>1$. 
It is important to note that every convex domain of polyhedral type satisfies conditions $\mathbb{C} 1$ and $\mathbb{C} 2$.

If $\Omega$ satisfies conditions $\mathbb{C} 1$ and $\mathbb{C} 2$ then using (2.3)-2.7) one has the following bound

$$
\left|\partial_{x_{j}} G(x, \xi)\right| \leq c|x-\xi|^{-2} \quad \text { and } \quad\left|\partial_{x_{j}} \partial_{\xi_{k}} G(x, \xi)\right| \leq c|x-\xi|^{-3}
$$

for all $x, \xi \in \Omega, j, k=1,2,3$. In particular, if $\Omega$ is a convex polyhedral domain the above estimate holds. In fact, by [12, 15, these estimates are valid for general convex domains.

\section{HÖLDER ESTIMATES OF GREEN'S FUnCTION}

Lemma 3.1. Let $\Omega \subset \mathbb{R}^{3}$ satisfy conditions $\mathbb{C} 1$ and $\mathbb{C} 2$ and let $m$ be an arbitrary positive number. Then the estimates (1.4) are satisfied with arbitrary $\sigma \in(0,1)$ for $|x-\xi|<m|x-y|$.

Proof. If $|x-\xi|<m|x-y|$, then $|y-\xi|<(m+1)|x-y|$ and 2.8) implies

$$
\begin{aligned}
& \frac{\left|\partial_{x_{i}} G(x, \xi)-\partial_{y_{i}} G(y, \xi)\right|}{|x-y|^{\sigma}} \leq \frac{\left|\partial_{x_{i}} G(x, \xi)\right|}{|x-y|^{\sigma}}+\frac{\left|\partial_{y_{i}} G(y, \xi)\right|}{|x-y|^{\sigma}} \\
& \leq c \frac{|x-\xi|^{-2}}{|x-y|^{\sigma}}+\frac{|y-\xi|^{-2}}{|x-y|^{\sigma}} \leq c(m+1)^{\sigma}\left(|x-\xi|^{-2-\sigma}+|y-\xi|^{-2-\sigma}\right) .
\end{aligned}
$$

In the same way, the second estimate of 1.4 holds.

In the following, we assume that $m$ is a sufficiently large positive number.

Lemma 3.2. Let $\Omega \subset \mathbb{R}^{3}$ satisfy conditions $\mathbb{C} 1$ and $\mathbb{C} 2$. Furthermore, let $\sigma$ be a positive number, $\sigma<1, \sigma<\Lambda_{j}-1$ for all $j$, and $\sigma<\mu_{k}-1$ for all $k$. Then there exists a constant $c$ such that

$$
\begin{aligned}
\frac{\left|\partial_{x_{i}} G(x, \xi)-\partial_{y_{i}} G(y, \xi)\right|}{|x-y|^{\sigma}} & \leq c|x-\xi|^{-2-\sigma}, \\
\frac{\left|\partial_{x_{i}} \partial_{\xi_{j}} G(x, \xi)-\partial_{y_{i}} \partial_{\xi_{j}} G(y, \xi)\right|}{|x-y|^{\sigma}} & \leq c|x-\xi|^{-3-\sigma} .
\end{aligned}
$$

for all $x, y, \xi \in \Omega, x \neq y,|x-\xi|>m|x-y|>r(x)$.

Proof. Since $|x-\xi|>m|x-y|$ with a sufficiently large $m$, we may assume that $x$ and $y$ lie in a neighborhood $\mathcal{V}_{j}$ of the same vertex $x^{(j)}$. As before we suppose that $\mathcal{V}_{j}$ has a positive distance to the edges $M_{k}, k \notin I_{j}$. From the condition $r(x)<m|x-y|$ it follows that

$$
r(y)<(m+1)|x-y| .
$$

Furthermore, the condition $|x-y|<|x-\xi| / m$ implies

$$
\left(1-\frac{1}{m}\right)|x-\xi|<|y-\xi|<\left(1+\frac{1}{m}\right)|x-\xi| .
$$

We consider the following cases

1) $\xi \in \mathcal{V}_{j} \quad$ and $\quad \rho_{j}(x)<\rho_{j}(\xi) / 2$

2) $\xi \in \mathcal{V}_{j} \quad$ and $\quad \rho_{j}(x)>2 \rho_{j}(\xi)$

3) $\xi \in \mathcal{V}_{j}$ and $\rho_{j}(\xi) / 2<\rho_{j}(x)<2 \rho_{j}(\xi)$

4) $\xi$ lies in a neighborhood of another vertex $x^{(\nu)}$ and $|x-\xi|>\delta$, where $\delta$ is a fixed positive number. 
We start with case 1$)$. Then obviously $|x-\xi|<\rho_{j}(x)+\rho_{j}(\xi)<\frac{3}{2} \rho_{j}(\xi)$ and

$$
\rho_{j}(y)<\rho_{j}(x)+|x-y|<\rho_{j}(x)+\frac{1}{m}|x-\xi|<\left(\frac{1}{2}+\frac{3}{2 m}\right) \rho_{j}(\xi) .
$$

Consequently (2.4) yields

$$
\begin{aligned}
\mid \partial_{x_{i}} G(x, \xi)- & \partial_{y_{i}} G(y, \xi)|\leq| \partial_{x_{i}} G(x, \xi)|+| \partial_{y_{i}} G(y, \xi) \mid \\
& \leq c \rho_{j}(\xi)^{-1-\Lambda_{j}+\varepsilon}\left(\rho_{j}(x)^{\Lambda_{j}-1-\varepsilon}\left(\frac{r(x)}{\rho_{j}(x)}\right)^{\sigma}+\rho_{j}(y)^{\Lambda_{j}-1-\varepsilon}\left(\frac{r(y)}{\rho_{j}(y)}\right)^{\sigma}\right) .
\end{aligned}
$$

Here $\varepsilon$ can be chosen such that $\Lambda_{j}-1-\varepsilon-\sigma \geq 0$. Thus,

$$
\left|\partial_{x_{i}} G(x, \xi)-\partial_{y_{i}} G(y, \xi)\right| \leq c \rho_{j}(\xi)^{-2-\sigma}\left(r(x)^{\sigma}+r(y)^{\sigma}\right) \leq c^{\prime}|x-\xi|^{-2-\sigma}|x-y|^{\sigma} .
$$

Analogously, we obtain

$$
\begin{aligned}
\mid \partial_{x_{i}} \partial_{\xi_{l}} G(x, \xi) & -\partial_{y_{i}} \partial_{\xi_{l}} G(y, \xi) \mid \\
& \leq c \rho_{j}(\xi)^{-2-\Lambda_{j}+\varepsilon}\left(\rho_{j}(x)^{\Lambda_{j}-1-\varepsilon}\left(\frac{r(x)}{\rho_{j}(x)}\right)^{\sigma}+\rho_{j}(y)^{\Lambda_{j}-1-\varepsilon}\left(\frac{r(y)}{\rho_{j}(y)}\right)^{\sigma}\right) \\
& \leq c \rho_{j}(\xi)^{-3-\sigma}\left(r(x)^{\sigma}+r(y)^{\sigma}\right) \leq c^{\prime}|x-\xi|^{-3-\sigma}|x-y|^{\sigma} .
\end{aligned}
$$

Case 2): In this case $|x-\xi|<\rho_{j}(x)+\rho_{j}(\xi)<\frac{3}{2} \rho_{j}(x)$ and

$$
\begin{aligned}
\rho_{j}(y) & >\rho_{j}(x)-|x-y|>\rho_{j}(x)-\frac{1}{m}|x-\xi|>\rho_{j}(x)-\frac{1}{m}\left(\rho_{j}(x)+\rho_{j}(\xi)\right) \\
& >\left(1-\frac{3}{2 m}\right) \rho_{j}(x)>\left(2-\frac{3}{m}\right) \rho_{j}(\xi) .
\end{aligned}
$$

Therefore by 2.3

$$
\begin{aligned}
\mid \partial_{x_{i}} G(x, \xi) & -\partial_{y_{i}} G(y, \xi)|\leq| \partial_{x_{i}} G(x, \xi)|+| \partial_{y_{i}} G(y, \xi) \mid \\
& \leq c \rho_{j}(\xi)^{\Lambda_{j}-\varepsilon}\left(\rho_{j}(x)^{-2-\Lambda_{j}+\varepsilon}\left(\frac{r(x)}{\rho_{j}(x)}\right)^{\sigma}+\rho_{j}(y)^{-2-\Lambda_{j}+\varepsilon}\left(\frac{r(y)}{\rho_{j}(y)}\right)^{\sigma}\right) \\
& \leq c^{\prime}\left(\rho_{j}(x)^{-2-\sigma} r(x)^{\sigma}+\rho_{j}(y)^{-2-\sigma} r(y)^{\sigma}\right) \\
& \leq c^{\prime \prime}|x-\xi|^{-2-\sigma}|x-y|^{\sigma}
\end{aligned}
$$

and analogously,

$$
\begin{aligned}
\mid \partial_{x_{i}} \partial_{\xi_{j}} G(x, \xi) & -\partial_{y_{i}} \partial_{\xi_{j}} G(y, \xi)|\leq| \partial_{x_{i}} \partial_{\xi_{j}} G(x, \xi)|+| \partial_{y_{i}} \partial_{\xi_{j}} G(y, \xi) \mid \\
& \leq c \rho_{j}(\xi)^{\Lambda_{j}-1-\varepsilon}\left(\rho_{j}(x)^{-2-\Lambda_{j}+\varepsilon}\left(\frac{r(x)}{\rho_{j}(x)}\right)^{\sigma}+\rho_{j}(y)^{-2-\Lambda_{j}+\varepsilon}\left(\frac{r(y)}{\rho_{j}(y)}\right)^{\sigma}\right) \\
& \leq c^{\prime}\left(\rho_{j}(x)^{-3-\sigma} r(x)^{\sigma}+\rho_{j}(y)^{-3-\sigma} r(y)^{\sigma}\right) \leq c^{\prime \prime}|x-\xi|^{-3-\sigma}|x-y|^{\sigma} .
\end{aligned}
$$

Case 3): Then $|x-\xi|<3 \rho_{j}(\xi)$ and

$$
\left(\frac{1}{2}-\frac{3}{m}\right) \rho_{j}(\xi)<\rho_{j}(y)<\left(2+\frac{3}{m}\right) \rho_{j}(\xi) .
$$

Since $r(x)<|x-\xi|$ and

$$
r(y)<(m+1)|x-y|<\frac{m+1}{m}|x-\xi|<\frac{m+1}{m-1}|y-\xi|,
$$


we can apply 2.5 and obtain

$$
\begin{aligned}
\left|\partial_{x_{i}} G(x, \xi)-\partial_{y_{i}} G(y, \xi)\right| & \leq\left|\partial_{x_{i}} G(x, \xi)\right|+\left|\partial_{y_{i}} G(y, \xi)\right| \\
& \leq c\left(|x-\xi|^{-2}\left(\frac{r(x)}{|x-\xi|}\right)^{\sigma}+|y-\xi|^{-2}\left(\frac{r(y)}{|y-\xi|}\right)^{\sigma}\right) \\
& \leq c^{\prime}|x-\xi|^{-2-\sigma}|x-y|^{\sigma} .
\end{aligned}
$$

Analogously,

$$
\begin{aligned}
\left|\partial_{x_{i}} \partial_{\xi_{l}} G(x, \xi)-\partial_{y_{i}} \partial_{\xi_{l}} G(y, \xi)\right| & \leq c\left(|x-\xi|^{-3}\left(\frac{r(x)}{|x-\xi|}\right)^{\sigma}+|y-\xi|^{-3}\left(\frac{r(y)}{|y-\xi|}\right)^{\sigma}\right) \\
& \leq c^{\prime}|x-\xi|^{-3-\sigma}|x-y|^{\sigma} .
\end{aligned}
$$

Case 4): Finally, we consider the case when $x$ and $y$ lie in the neighborhood $\mathcal{V}_{j}$ of the vertex $x^{(j)}$ and $\xi$ lies in a neighborhood of another vertex $x^{(\nu)}$ such that $|x-\xi|>\delta$, where $\delta$ is a fixed positive number. Then by (2.7)

$$
\begin{aligned}
\mid \partial_{x_{i}} G(x, \xi) & -\partial_{y_{i}} G(y, \xi)|\leq| \partial_{x_{i}} G(x, \xi)|+| \partial_{y_{i}} G(y, \xi) \mid \\
& \leq c \rho_{\nu}(\xi)^{\Lambda_{\nu}-\varepsilon}\left(\rho_{j}(x)^{\Lambda_{j}-1-\varepsilon}\left(\frac{r(x)}{\rho_{j}(x)}\right)^{\sigma}+\rho_{j}(y)^{\Lambda_{j}-1-\varepsilon}\left(\frac{r(y)}{\rho_{j}(y)}\right)^{\sigma}\right) \\
& \leq c^{\prime}\left(r(x)^{\sigma}+r(y)^{\sigma}\right) \leq 2 c^{\prime}(m+1)^{\sigma}|x-y|^{\sigma}
\end{aligned}
$$

and analogously,

$$
\begin{aligned}
\mid \partial_{x_{i}} \partial_{\xi_{l}} G(x, \xi) & -\partial_{y_{i}} \partial_{\xi_{l}} G(y, \xi)|\leq| \partial_{x_{i}} \partial_{\xi_{l}} G(x, \xi)|+| \partial_{y_{i}} \partial_{\xi_{l}} G(y, \xi) \mid \\
& \leq c \rho_{\nu}(\xi)^{\Lambda_{\nu}-1-\varepsilon}\left(\rho_{j}(x)^{\Lambda_{j}-1-\varepsilon}\left(\frac{r(x)}{\rho_{j}(x)}\right)^{\sigma}+\rho_{j}(y)^{\Lambda_{j}-1-\varepsilon}\left(\frac{r(y)}{\rho_{j}(y)}\right)^{\sigma}\right) \\
& \leq c^{\prime}|x-y|^{\sigma} .
\end{aligned}
$$

This completes the proof.

Lemma 3.3. Let $\Omega \subset \mathbb{R}^{3}$ be a convex domain of polyhedral type. Furthermore, let $\sigma$ be a positive number, $\sigma<\Lambda_{j}-1$ for all $j, \sigma<\mu_{k}-1$ for all $k$, and $\sigma<1$. Then there exists a constant $c$ such that the estimates (3.1) and (3.2) are satisfied for all $x, y, \xi \in \Omega, \xi \neq x \neq y,|x-\xi|>m|x-y|, r(x)>m|x-y|$.

Proof. From the mean value theorem it follows that

$$
\frac{\left|\partial_{x_{i}} G(x, \xi)-\partial_{y_{i}} G(y, \xi)\right|}{|x-y|^{\sigma}} \leq|x-y|^{1-\sigma}\left|\nabla_{z} \partial_{z_{i}} G(z, \xi)\right|
$$

and

$$
\frac{\left|\partial_{x_{i}} \partial_{\xi_{l}} G(x, \xi)-\partial_{y_{i}} \partial_{\xi_{l}} G(y, \xi)\right|}{|x-y|^{\sigma}} \leq|x-y|^{1-\sigma}\left|\nabla_{z} \partial_{z_{i}} \partial_{\xi_{l}} G(z, \xi)\right|,
$$

where $z=x+t(y-x), 0<t<1$. Since $\Omega$ is convex $z \in \Omega$. We assume again that $x$ and $y$ lie in the neighborhood $\mathcal{V}_{j}$ of the vertex $x^{(j)}$ and consider the same cases 1)-4) as in the proof of Lemma 3.2 .

Case 1): Since

$$
\rho_{j}(z)<\rho_{j}(x)+|x-y|<\rho_{j}(x)+\frac{1}{m}|x-\xi|<\rho_{j}(x)+\frac{1}{m}\left(\rho_{j}(x)+\rho_{j}(\xi)\right)<\left(\frac{1}{2}+\frac{3}{2 m}\right) \rho_{j}(\xi),
$$


the derivatives of $G$ at the point $(z, \xi)$ satisfy the estimates (cf. 2.4p)

$$
\left|\nabla_{z} \partial_{z_{i}} G(z, \xi)\right| \leq \rho_{j}(z)^{\Lambda_{j}-2-\varepsilon} \rho_{j}(\xi)^{-1-\Lambda_{j}+\varepsilon}\left(\frac{r(z)}{\rho_{j}(z)}\right)^{\sigma-1}
$$

and

$$
\left|\nabla_{z} \partial_{z_{i}} \partial_{\xi_{l}} G(z, \xi)\right| \leq c \rho_{j}(z)^{\Lambda_{j}-2-\varepsilon} \rho_{j}(\xi)^{-2-\Lambda_{j}+\varepsilon}\left(\frac{r(z)}{\rho_{j}(z)}\right)^{\sigma-1} .
$$

The number $\varepsilon$ can be chosen such that $\Lambda_{j}-1-\varepsilon-\sigma \geq 0$. Consequently,

$$
\frac{\left|\partial_{x_{i}} G(x, \xi)-\partial_{y_{i}} G(y, \xi)\right|}{|x-y|^{\sigma}} \leq c|x-y|^{1-\sigma} \rho_{j}(\xi)^{-2-\sigma} r(z)^{\sigma-1}
$$

and

$$
\frac{\left|\partial_{x_{i}} \partial_{\xi_{l}} G(x, \xi)-\partial_{y_{i}} \partial_{\xi_{l}} G(y, \xi)\right|}{|x-y|^{\sigma}} \leq|x-y|^{1-\sigma} \rho_{j}(\xi)^{-3-\sigma} r(z)^{\sigma-1} .
$$

Using the inequalities $r(z)>(m-1)|x-y|$ and $\rho_{j}(\xi)>\frac{2}{3}|x-\xi|$, we get $(3.1)$ and 3.2 .

In Case 2) we obtain the estimate

$$
\begin{aligned}
\rho_{j}(z) & >\rho_{j}(x)-|x-y|>\rho_{j}(x)-\frac{1}{m}|x-\xi|>\rho_{j}(x)-\frac{1}{m}\left(\rho_{j}(x)+\rho_{j}(\xi)\right) \\
& >\left(1-\frac{3}{2 m}\right) \rho_{j}(x)>\left(2-\frac{3}{m}\right) \rho_{j}(\xi) .
\end{aligned}
$$

Therefore by 2.3

$$
\left|\nabla_{z} \partial_{z_{i}} G(z, \xi)\right| \leq c \rho_{j}(z)^{-3-\Lambda_{j}+\varepsilon} \rho_{j}(\xi)^{\Lambda_{j}-\varepsilon}\left(\frac{r(z)}{\rho_{j}(z)}\right)^{\sigma-1} \leq c^{\prime} \rho_{j}(z)^{-2-\sigma} r(z)^{\sigma-1},
$$

and

$$
\left|\nabla_{z} \partial_{z_{i}} \partial_{\xi_{l}} G(z, \xi)\right| \leq c \rho_{j}(z)^{-3-\Lambda_{j}+\varepsilon} \rho_{j}(\xi)^{\Lambda_{j}-1-\varepsilon}\left(\frac{r(z)}{\rho_{j}(z)}\right)^{\sigma-1} \leq c^{\prime} \rho_{j}(z)^{-3-\sigma} r(z)^{\sigma-1},
$$

Using the inequalities $r(z)>(m-1)|x-y|$ and

$$
\rho_{j}(z)>\rho_{j}(x)-|x-y|>\frac{2}{3}|x-\xi|-\frac{1}{m}|x-\xi|,
$$

we obtain (3.1) and 3.2 .

Case 3): From the inequalities $\rho_{j}(\xi) / 2<\rho_{j}(x)<2 \rho_{j}(\xi)$ and $|x-\xi|>m|x-z|$ it follows that

$$
\left(\frac{1}{2}-\frac{3}{m}\right) \rho_{j}(\xi)<\rho_{j}(z)<\left(2+\frac{3}{m}\right) \rho_{j}(\xi) .
$$

Furthermore, the inequalities $|x-\xi|>m|x-z|$ and $r(x)>m|x-z|$ yield (3.5)

$\left(1-\frac{1}{m}\right)|x-\xi|<|z-\xi|<\left(1+\frac{1}{m}\right)|x-\xi| \quad$ and $\quad\left(1-\frac{1}{m}\right) r(x)<r(z)<\left(1+\frac{1}{m}\right) r(x)$.

If $|z-\xi|>\min (r(z), r(\xi))$, then $(2.5)$ and (3.5) imply

$$
\begin{aligned}
\left|\nabla_{z} \partial_{z_{i}} G(z, \xi)\right| \leq c|z-\xi|^{-3}\left(\frac{r(z)}{|z-\xi|}\right)^{\sigma-1} & \leq c^{\prime}|x-\xi|^{-2-\sigma} r(x)^{\sigma-1} \\
& \leq c^{\prime} m^{\sigma-1}|x-\xi|^{-2-\sigma}|x-y|^{\sigma-1} .
\end{aligned}
$$

In the case $|z-\xi|<\min (r(z), r(\xi))$, it follows from 2.6) that

$$
\left|\nabla_{z} \partial_{z_{i}} G(z, \xi)\right| \leq c|z-\xi|^{-3} \leq c^{\prime}|x-\xi|^{-3} \leq c^{\prime} m^{\sigma-1}|x-\xi|^{-2-\sigma}|x-y|^{\sigma-1} .
$$


This together with (3.3) implies (3.1). Analogously, we obtain the estimates

$$
\left|\nabla_{z} \partial_{z_{i}} \partial_{\xi_{l}} G(z, \xi)\right| \leq c|z-\xi|^{-4}\left(\frac{r(z)}{|z-\xi|}\right)^{\sigma-1} \leq c^{\prime}|x-\xi|^{-3-\sigma}|x-y|^{\sigma-1}
$$

for $|z-\xi|>\min (r(z), r(\xi))$ and

$$
\left|\nabla_{z} \partial_{z_{i}} \partial_{\xi_{l}} G(z, \xi)\right| \leq c|z-\xi|^{-4} \leq c^{\prime} m^{\sigma-1}|x-\xi|^{-3-\sigma}|x-y|^{\sigma-1} .
$$

for $|z-\xi|<\min (r(z), r(\xi))$ what together with (3.4) yields 3.2 .

Case 4): Suppose that $\xi$ lies in a neighborhood $\mathcal{V}_{\nu}$ of the vertex $x^{(\nu)}$ and that $|x-\xi|>\delta$, where $\delta$ is a fixed positive number. Then

$$
\left|\nabla_{z} \partial_{z_{i}} G(z, \xi)\right| \leq c \rho_{j}(z)^{\Lambda_{j}-2-\varepsilon} \rho_{\nu}(\xi)^{\Lambda_{\nu}-\varepsilon}\left(\frac{r(z)}{\rho_{j}(z)}\right)^{\sigma-1} \leq c^{\prime} r(z)^{\sigma-1}
$$

and

$$
\left|\nabla_{z} \partial_{z_{i}} \partial_{\xi_{l}} G(z, \xi)\right| \leq c \rho_{j}(z)^{\Lambda_{j}-2-\varepsilon} \rho_{\nu}(\xi)^{\Lambda_{\nu}-1-\varepsilon}\left(\frac{r(z)}{\rho_{j}(z)}\right)^{\sigma-1} \leq r(z)^{\sigma-1} .
$$

Using the last two estimates together with the inequalities 3.3$), 3.4,|x-\xi|>\delta$ and $r(z)>(m-1)|x-y|$, we obtain 3.1) and 3.2. The proof of the lemma is complete.

Now the following theorem holds as an immediate consequence of Lemmas 3.1 3.3 .

Theorem 1. Let $\Omega \subset \mathbb{R}^{3}$ be a convex domain of polyhedral type. Furthermore, let $\sigma$ be a positive number, $\sigma<1, \sigma<\Lambda_{j}-1$ for all $j$, and $\sigma<\mu_{k}-1$ for all $k$. Then there exists a finite constant $c$ such that the estimates (1.4) are satisfied for all $x, y, \xi \in \Omega, x \neq y$.

As an example, we consider the case when $\Omega$ is a cube. In this case, $\Lambda_{j}=3$ for all $j$ and $\mu_{k}=2$ for all $k$. Consequently, the estimates (1.4) are valid for arbitrary $0<\sigma<1$.

Remark 1. In the proof of Theorem 1, we used only the estimates of Green's function in Section 2.3 and the fact that $\Lambda_{j}>1$ and $\mu_{k}>1$ for convex domains of polyhedral type. The result of the theorem is also true for other second order elliptic equations or systems provided the Green's function (matrix) satisfies the estimates $(2.3)-(2.7)$ with exponents $\Lambda_{j}$ and $\mu_{k}$ greater than 1. For example, the estimates (1.4) hold for the Green matrix of the Dirichlet problem to the Lamè system.

\section{Applichtions to Finite Element Methods.}

4.1. Preliminaries and basic assumptions. For the finite element approximation of the problem, let $\left\{\mathcal{T}_{h}\right\}_{h}, 0<h<1$, be a sequence of triangulations of $\Omega$, $\bar{\Omega}=\bigcup_{\tau \in \mathcal{T}_{h}} \bar{\tau}$, with the elements $\tau$ mutually disjoint. The partitions are face-to-face so that simplices meet only in full lower-dimensional faces or not at all. The triangulations are assumed to be quasi-uniform, i.e. (if necessary after a renormalization of $h$ ),

$$
\operatorname{diam} \tau \leq h \leq C(\text { meas } \tau)^{1 / 3}, \quad \forall \tau \in \mathcal{T}_{h} .
$$

Our finite element spaces are then the $\mathcal{C}^{0}$ simplicial Lagrange elements

$$
S_{h}=S_{h}^{k}(\Omega)=\left\{\chi \in H_{0}^{1}(\Omega):\left.v\right|_{\tau} \in \mathbb{P}^{k}(\tau), \forall \tau \in \mathcal{T}_{h}\right\},
$$


where $\mathbb{P}^{k}(\tau)$ denotes the set of polynomials of degree less than or equal to $k$ on $\tau$. Thus the scaling properties hold.

The particular approximation property we actually need is a standard approximation result (cf. [10]).

Lemma 4.1. Let $D \subset D_{d} \subset \Omega$, with $d \geq \kappa h$, for some fixed $\kappa$ sufficiently large and let $D_{d}=\{x \in \Omega: \operatorname{dist}(x, \partial D) \leq d\}$. Furthermore, let $I^{h}: C(\Omega) \rightarrow S_{h}$ be the Lagrange interpolant. For any $v \in C^{1+\sigma}(\Omega)$ there exists $C$ independent of $h$ such that

$$
\left\|v-I^{h} v\right\|_{W_{\infty}^{t}(D)} \leq C h^{1-t+\sigma}\|v\|_{C^{1+\sigma}\left(D_{d}\right)}, \quad t=0,1,
$$

where

$$
\|v\|_{C^{1+\sigma}(D)}=\|v\|_{C^{1}(D)}+\sup _{x_{1}, x_{2} \in D} \frac{\left|\nabla v\left(x_{1}\right)-\nabla v\left(x_{2}\right)\right|}{\left|x_{1}-x_{2}\right|^{\sigma}}
$$

Another result that we need is the local energy error estimate (cf. [26]):

Lemma 4.2. Let $D \subset D_{d} \subset \Omega$, with $d \geq \kappa h$, for some fixed $\kappa$ sufficiently large and let $D_{d}=\{x \in \Omega: \operatorname{dist}(x, \partial D \backslash \partial \Omega) \leq d\}$. If $w \in H_{0}^{1}(\Omega)$ and $w_{h} \in S_{h}$ satisfy

$$
\left(\nabla\left(w-w_{h}\right), \nabla \chi\right)_{\Omega}=0, \quad \forall \chi \in S_{h},
$$

then

$$
\begin{aligned}
\left\|w-w_{h}\right\|_{H^{1}(D)} \leq & C \min _{\chi \in S_{h}}\left(\|w-\chi\|_{H^{1}\left(D_{d}\right)}+d^{-1}\|w-\chi\|_{L^{2}\left(D_{d}\right)}\right) \\
& +C d^{-1}\left\|w-w_{h}\right\|_{L^{2}\left(D_{d}\right)},
\end{aligned}
$$

where $C$ is independent of $D, h, d, w$, and $w_{h}$.

\subsection{Best Approximation Result.}

Theorem 2. Let $\Omega \subset \mathbb{R}^{3}$ be a convex polyhedral domain and suppose that u satisfies (1.1) and let $u_{h} \in S_{h}$ be its finite element approximation. Then, there exists a constant $C$ independent of $h, u$ and $u_{h}$ such that 1.2 holds.

Proof. Let $z \in \Omega$ and $z \in \tau$ for some $\tau \in \mathcal{T}_{h}$. We will be interested in bounding $\left|\nabla\left(u-u_{h}\right)(z)\right|$. There exists $\eta \in C_{c}^{1}(\tau)$, the regularized Dirac delta function, which satisfies

$$
\int_{\tau} \chi \eta=(\chi, \eta)_{\tau}=\chi(z), \quad \forall \chi \in \mathbb{P}^{k}(\tau)
$$

with the property

$$
\left\|D^{s} \eta\right\|_{L^{\infty}(\Omega)} \leq C h^{-3-t}, \text { for } t=0,1 .
$$

Let $\partial_{z_{l}}\left(u-u_{h}\right)$ denote one of the partial derivatives of $u-u_{h}$. Now we define the function $g$, which satisfies the following equation,

$$
(\nabla g, \nabla \phi)=\left(\partial_{z_{l}} \eta, \phi\right), \quad \forall \phi \in H_{0}^{1}(\Omega) .
$$


Then for any $\chi \in S_{h}$ using 4.5 and the Galerkin orthogonality,

$$
\begin{aligned}
\partial_{z_{l}}\left(\chi-u_{h}\right)(z) & =\left(\partial_{z_{l}}\left(\chi-u_{h}\right), \eta\right)=-\left(\chi-u_{h}, \partial_{z_{l}} \eta\right) \\
& =-\left(\nabla\left(\chi-u_{h}\right), \nabla g\right) \\
& =\left(\nabla\left(u_{h}-u\right), \nabla g\right)+(\nabla(u-\chi), \nabla g) \\
& =\left(\nabla\left(u_{h}-u\right), \nabla\left(g-g_{h}\right)\right)+\left(u-\chi, \partial_{z_{l}} \eta\right) \\
& =\left(\nabla(\chi-u), \nabla\left(g-g_{h}\right)\right)-\left(\partial_{z_{l}}(u-\chi), \eta\right) \\
& \leq\|\nabla(u-\chi)\|_{L^{\infty}(\Omega)}\left(\|\eta\|_{L^{1}(\Omega)}+\left\|\nabla\left(g-g_{h}\right)\right\|_{L^{1}(\Omega)}\right) \\
& \leq\|\nabla(u-\chi)\|_{L^{\infty}(\Omega)}\left(C+\left\|\nabla\left(g-g_{h}\right)\right\|_{L^{1}(\Omega)}\right) .
\end{aligned}
$$

Hence,

$$
\left|\partial_{z_{l}}\left(u-u_{h}\right)(z)\right| \leq\|\nabla(u-\chi)\|_{L^{\infty}(\Omega)}\left(C+\left\|\nabla\left(g-g_{h}\right)\right\|_{L^{1}(\Omega)}\right) .
$$

Since the above estimate is valid for arbitrary $\chi \in S_{h}$ and any partial derivative, taking the infimum over $S_{h}$ and supremum over all partial derivatives, we obtain

$$
\left|\nabla\left(u-u_{h}\right)(z)\right| \leq \inf _{\chi \in S_{h}}\|\nabla(u-\chi)\|_{L^{\infty}(\Omega)}\left(C+\left\|\nabla\left(g-g_{h}\right)\right\|_{L^{1}(\Omega)}\right) .
$$

Thus, in order to establish the estimate 1.2 , we need to show

$$
\left\|\nabla\left(g-g_{h}\right)\right\|_{L^{1}(\Omega)} \leq C .
$$

Note, $g$ depends on $z$. Thus, we shall prove the above inequality with constant $C$ independent of $z$. We prove 4.6 in several steps.

Step 1: Dyadic decomposition Without loss of generality we assume that the diameter of $\Omega$ is less than 1 . We use a dyadic decomposition of $\Omega$. Let $d_{j}=2^{-j}$ then we have

$$
\Omega=\Omega^{*} \cup \bigcup_{j=0}^{J} \Omega_{j}
$$

where

$$
\begin{aligned}
& \Omega^{*}=\{x \in \Omega:|x-z| \leq K h\}, \\
& \Omega_{j}=\left\{x \in \Omega: d_{j+1} \leq|x-z| \leq d_{j}\right\},
\end{aligned}
$$

where $K$ is a sufficiently large constant to be chosen later and $J$ is the smallest integer such that $2^{-J} \geq K h$. Note that $J \approx|\log h|$. In the analysis below the generic constants will be denoted by $C$, but we will keep track on the explicit dependence of the constants on $K$. This will be important later for the double kickback argument.

Thus, by the Cauchy-Schwarz inequality, we have

$$
\left\|\nabla\left(g-g_{h}\right)\right\|_{L^{1}(\Omega)} \leq C K^{3 / 2} h^{3 / 2}\left\|\nabla\left(g-g_{h}\right)\right\|_{L^{2}\left(\Omega^{*}\right)}+C \sum_{j=0}^{J} d_{j}^{3 / 2}\left\|\nabla\left(g-g_{h}\right)\right\|_{L^{2}\left(\Omega_{j}\right)} .
$$

First we estimate the first term. Using the Cauchy-Schwarz inequality, the global a priori error estimates, (4.4), and $H^{2}$ regularity we have

$$
\begin{aligned}
(K h)^{3 / 2}\left\|\nabla\left(g-g_{h}\right)\right\|_{L^{2}\left(\Omega^{*}\right)} & \leq C K^{3 / 2} h^{3 / 2+1}\left\|D^{2} g\right\|_{L^{2}(\Omega)} \\
& \leq C K^{3 / 2} h^{5 / 2}\|\nabla \eta\|_{L^{2}(\tau)} \\
& \leq C K^{3 / 2} h^{4}\|\nabla \eta\|_{L^{\infty}(\tau)} \leq C K^{3 / 2} .
\end{aligned}
$$


Thus, we have

$$
\left\|\nabla\left(g-g_{h}\right)\right\|_{L^{1}(\Omega)} \leq C K^{3 / 2}+\sum_{j=0}^{J} M_{j} .
$$

where

$$
M_{j}=d_{j}^{3 / 2}\left\|\nabla\left(g-g_{h}\right)\right\|_{L^{2}\left(\Omega_{j}\right)} .
$$

Step 2: Initial Estimate for $M_{j}$. We first need to define the following sets

$$
\begin{aligned}
\Omega_{j}^{\prime} & =\left\{x \in \Omega: d_{j+2} \leq|x-z| \leq d_{j-1}\right\}, \\
\Omega_{j}^{\prime \prime} & =\left\{x \in \Omega: d_{j+3} \leq|x-z| \leq d_{j-2}\right\} .
\end{aligned}
$$

By the local energy estimate 4.3 ,

$$
\begin{aligned}
\left\|\nabla\left(g-g_{h}\right)\right\|_{L^{2}\left(\Omega_{j}\right)} \leq & C\left(\left\|\nabla\left(g-I^{h} g\right)\right\|_{L^{2}\left(\Omega_{j}^{\prime}\right)}+d_{j}^{-1}\left\|g-I^{h} g\right\|_{L^{2}\left(\Omega_{j}^{\prime}\right)}\right. \\
& \left.+d_{j}^{-1}\left\|g-g_{h}\right\|_{L^{2}\left(\Omega_{j}^{\prime}\right)}\right) .
\end{aligned}
$$

First we will treat the first two terms on the right hand side. By the CauchySchwarz inequality and the approximation result (4.1)

$$
\begin{aligned}
& \left\|\nabla\left(g-I^{h} g\right)\right\|_{L^{2}\left(\Omega_{j}^{\prime}\right)}+d_{j}^{-1}\left\|g-I^{h} g\right\|_{L^{2}\left(\Omega_{j}^{\prime}\right)} \\
& \leq C d_{j}^{3 / 2}\left(\left\|\nabla\left(g-I^{h} g\right)\right\|_{L^{\infty}\left(\Omega_{j}^{\prime}\right)}+d_{j}^{-1}\left\|g-I^{h} g\right\|_{L^{\infty}\left(\Omega_{j}^{\prime}\right)}\right) \\
& \leq C d_{j}^{3 / 2} h^{\sigma}\|g\|_{C^{1+\sigma}\left(\Omega_{j}^{\prime \prime}\right)} .
\end{aligned}
$$

Now we will use the Hölder estimates (1.4) to derive a bound for $\|g\|_{C^{1+\sigma}\left(\Omega_{j}^{\prime \prime}\right)}$. Using the Green's function representation we have,

$$
\begin{aligned}
\partial_{x_{i}} g(x)-\partial_{y_{i}} g(y) & =-\int_{\Omega}\left(\partial_{x_{i}} G(x, \xi)-\partial_{y_{i}} G(y, \xi)\right) \partial_{\xi_{l}} \eta(\xi) d \xi \\
& =\int_{\tau}\left(\partial_{\xi_{l}} \partial_{x_{i}} G(x, \xi)-\partial_{\xi_{l}} \partial_{y_{i}} G(y, \xi)\right) \eta(\xi) d \xi, \quad i=1,2,3 .
\end{aligned}
$$

Let $x, y \in \Omega_{j}^{\prime \prime}, x \neq y$, then by 1.4 ,

$$
\begin{aligned}
\frac{\left|\partial_{x_{i}} g(x)-\partial_{y_{i}} g(y)\right|}{|x-y|^{\sigma}} & \leq \max _{\xi \in \tau} \frac{\left|\partial_{\xi_{l}} \partial_{x_{i}} G(x, \xi)-\partial_{\xi_{l}} \partial_{y_{i}} G(y, \xi)\right|}{|x-y|^{\sigma}}\|\eta\|_{L^{1}(\tau)} \\
& \leq C \max _{\xi \in \tau}\left(|x-\xi|^{-3-\sigma}+|y-\xi|^{-3-\sigma}\right)\|\eta\|_{L^{1}(\tau)} \leq d_{j}^{-3-\sigma} .
\end{aligned}
$$

In the last inequality we used that for any $\xi \in \tau,|x-\xi|,|y-\xi| \geq C d_{j}$ and $\|\eta\|_{L^{1}(\tau)} \leq C$. Therefore,

$$
\sup _{x, y \in \Omega_{j}^{\prime \prime}} \frac{|\nabla g(x)-\nabla g(y)|}{|x-y|^{\sigma}} \leq d_{j}^{-3-\sigma} .
$$

Similarly, we can bound the other term of $\|g\|_{C^{1+\sigma}\left(\Omega_{j}^{\prime \prime}\right)}$ to obtain

$$
\|g\|_{C^{1+\sigma}\left(\Omega_{j}^{\prime \prime}\right)} \leq C d_{j}^{-3-\sigma} .
$$

Thus, we have shown that

$$
\left\|\nabla\left(g-I^{h} g\right)\right\|_{L^{2}\left(\Omega_{j}^{\prime}\right)}+d_{j}^{-1}\left\|g-I^{h} g\right\|_{L^{2}\left(\Omega_{j}^{\prime}\right)} \leq C d^{-3 / 2-\sigma} h^{\sigma} .
$$


Hence,

$$
M_{j} \leq C\left(h / d_{j}\right)^{\sigma}+d_{j}^{1 / 2}\left\|g-g_{h}\right\|_{L^{2}\left(\Omega_{j}^{\prime}\right)} .
$$

We still need to estimate $\left\|g-g_{h}\right\|_{L^{2}\left(\Omega_{j}^{\prime}\right)}$. We will accomplish it by a duality argument.

Step 3: Duality argument. We have the following representation

$$
\left\|g-g_{h}\right\|_{L^{2}\left(\Omega_{j}^{\prime}\right)}=\sup _{v \in C_{c}^{\infty}\left(\Omega_{j}^{\prime}\right),\|v\|_{L^{2}\left(\Omega_{j}^{\prime}\right)} \leq 1}\left(g-g_{h}, v\right) .
$$

Let $w$ be the solution of the following problem

$$
\begin{aligned}
-\Delta w=v, & & \text { in } \Omega, \\
w=0, & & \text { on } \partial \Omega .
\end{aligned}
$$

Thus, if $I^{h} w \in S_{h}$ denotes the interpolant of $w$, we have

$$
\begin{aligned}
\left(g-g_{h}, v\right) & =\left(\nabla\left(g-g_{h}\right), \nabla w\right)=\left(\nabla\left(g-g_{h}\right), \nabla\left(w-I^{h} w\right)\right) \\
& =\left(\nabla\left(g-g_{h}\right), \nabla\left(w-I^{h} w\right)\right)_{\Omega_{j}^{\prime \prime}}+\left(\nabla\left(g-g_{h}\right), \nabla\left(w-I^{h} w\right)\right)_{\Omega \backslash \Omega_{j}^{\prime \prime}} .
\end{aligned}
$$

First we estimate $\left(\nabla\left(g-g_{h}\right), \nabla\left(w-I^{h} w\right)\right)_{\Omega_{j}^{\prime \prime}}$. By the Cauchy-Schwarz inequality, the global a priori error estimate, and $H^{2}$ regularity we have

$$
\begin{aligned}
\left(\nabla\left(g-g_{h}\right), \nabla\left(w-I^{h} w\right)\right)_{\Omega_{j}^{\prime \prime}} & \leq\left\|\nabla\left(g-g_{h}\right)\right\|_{L^{2}\left(\Omega_{j}^{\prime \prime}\right)}\left\|\nabla\left(w-I^{h} w\right)\right\|_{L^{2}(\Omega)} \\
& \leq\left\|\nabla\left(g-g_{h}\right)\right\|_{L^{2}\left(\Omega_{j}^{\prime \prime}\right)} C h\left\|D^{2} w\right\|_{L^{2}(\Omega)} \\
& \leq C h\left\|\nabla\left(g-g_{h}\right)\right\|_{L^{2}\left(\Omega_{j}^{\prime \prime}\right)} .
\end{aligned}
$$

Next we estimate the second term

$$
\begin{aligned}
\left(\nabla\left(g-g_{h}\right), \nabla\left(w-I^{h} w\right)\right)_{\Omega \backslash \Omega_{j}^{\prime \prime}} & \leq\left\|\nabla\left(g-g_{h}\right)\right\|_{L^{1}(\Omega)}\left\|\nabla\left(w-I^{h} w\right)\right\|_{L^{\infty}\left(\Omega \backslash \Omega_{j}^{\prime \prime}\right)} \\
& \leq\left\|\nabla\left(g-g_{h}\right)\right\|_{L^{1}(\Omega)} C h^{\sigma}\|w\|_{C^{1+\sigma}\left(\Omega \backslash \Omega_{j}^{\prime \prime}\right)}
\end{aligned}
$$

Since $\Omega \backslash \Omega_{j}^{\prime \prime}$ is separated from $\Omega_{j}^{\prime}$ by at least $d_{j}$, we have for $x, y \in \Omega \backslash \Omega_{j}^{\prime \prime}$, using the first estimate of (1.4)

$$
\begin{aligned}
\frac{\left|\partial_{x_{i}} w(x)-\partial_{y_{i}} w(y)\right|}{|x-y|^{\sigma}} & \leq \int_{\Omega_{j}^{\prime}} \frac{\left|\partial_{x_{i}} G(x, \xi)-\partial_{y_{i}} G(y, \xi)\right|}{|x-y|^{\sigma}}|v(\xi)| d \xi \\
& \leq \max _{\xi \in \Omega_{j}^{\prime}}(|x-\xi|+|y-\xi|)^{-2-\sigma} \int_{\Omega_{j}^{\prime}}|v(\xi)| d \xi \\
& \leq C d_{j}^{-2-\sigma} d_{j}^{3 / 2}\|v\|_{L^{2}\left(\Omega_{j}^{\prime}\right)} \leq C d_{j}^{-1 / 2-\sigma} .
\end{aligned}
$$

Hence,

$$
\|w\|_{C^{1+\sigma}\left(\Omega \backslash \Omega_{j}^{\prime \prime}\right)} \leq C d_{j}^{-1 / 2-\sigma}
$$

which implies that

$$
\left(\nabla\left(g-g_{h}\right), \nabla\left(w-I^{h} w\right)\right)_{\Omega \backslash \Omega_{j}^{\prime \prime}} \leq C h^{\sigma} d_{j}^{-1 / 2-\sigma}\left\|\nabla\left(g-g_{h}\right)\right\|_{L^{1}(\Omega)} .
$$

Therefore,

$$
\left\|g-g_{h}\right\|_{L^{2}\left(\Omega_{j}^{\prime}\right)} \leq C h^{\sigma} d_{j}^{-1 / 2-\sigma}\left\|\nabla\left(g-g_{h}\right)\right\|_{L^{1}(\Omega)}+C h\left\|\nabla\left(g-g_{h}\right)\right\|_{L^{2}\left(\Omega_{j}^{\prime \prime}\right)} .
$$

To summarize,

$$
M_{j} \leq C\left(h / d_{j}\right)^{\sigma}+C\left(h / d_{j}\right)^{\sigma}\left\|\nabla\left(g-g_{h}\right)\right\|_{L^{1}(\Omega)}+C h d_{j}^{1 / 2}\left\|\nabla\left(g-g_{h}\right)\right\|_{L^{2}\left(\Omega_{j}^{\prime \prime}\right)} .
$$


Step 4: Double kick-back argument. Summing over $j$ we obtain

$$
\sum_{j=0}^{J} M_{j} \leq \frac{C}{K^{\sigma}}+\frac{C}{K^{\sigma}}\left\|\nabla\left(g-g_{h}\right)\right\|_{L^{1}(\Omega)}+\frac{C h}{d_{J}} \sum_{j=0}^{J} d_{j}^{3 / 2}\left\|\nabla\left(g-g_{h}\right)\right\|_{L^{2}\left(\Omega_{j}^{\prime \prime}\right)},
$$

where we have used that

$$
\sum_{j=0}^{J}\left(h / d_{j}\right)^{\sigma} \leq h^{\sigma} \sum_{j=0}^{J} 2^{j \sigma} \leq C h^{\sigma} 2^{\sigma J} \leq C K^{-\sigma} \quad \text { and } \quad d_{j}^{-1} \leq d_{J}^{-1} .
$$

Clearly,

$$
\begin{aligned}
\sum_{j=0}^{J} d_{j}^{3 / 2}\left\|\nabla\left(g-g_{h}\right)\right\|_{L^{2}\left(\Omega_{j}^{\prime \prime}\right)} & \leq C \sum_{j=0}^{J} M_{j}+C(K h)^{3 / 2}\left\|\nabla\left(g-g_{h}\right)\right\|_{L^{2}\left(\Omega^{*}\right)} \\
& \leq C \sum_{j=0}^{J} M_{j}+C K^{3 / 2} .
\end{aligned}
$$

Thus, using that $h / d_{J} \leq K^{-1}$, and taking $K$ large enough we have

$$
\sum_{j=0}^{J} M_{j} \leq C\left(K^{3 / 2}+1\right)+\frac{C}{K^{\sigma}}\left\|\nabla\left(g-g_{h}\right)\right\|_{L^{1}(\Omega)} .
$$

Therefore, if we plug this result into 4.7 we get

$$
\left\|\nabla\left(g-g_{h}\right)\right\|_{L^{1}(\Omega)} \leq C\left(K^{3 / 2}+1\right)+\frac{C}{K^{\sigma}}\left\|\nabla\left(g-g_{h}\right)\right\|_{L^{1}(\Omega)} .
$$

Again by choosing $K$ large enough we can conclude

$$
\left\|\nabla\left(g-g_{h}\right)\right\|_{L^{1}(\Omega)} \leq C .
$$

Thus the proof of (4.6) is complete and hence we have established (1.2).

\subsection{Localized Pointwise Error Estimate.}

Theorem 3. With the assumptions of Theorem 2, the following estimate holds,

$$
\left|\nabla\left(u-u_{h}\right)(z)\right| \leq C \inf _{\chi \in S_{h}}\left\|\omega^{s} \nabla(u-\chi)\right\|_{L^{\infty}(\Omega)},
$$

for any $0 \leq s<\sigma$, where $\omega=\omega_{z, h}(y)=\frac{h}{h+|z-y|}$ is the weight function.

The proof of this result is very similar to the proof of Theorem 2, We leave the details to the reader.

Following the presentation in [31, one can obtain the following error expansion inequality, which shows that the error is localized.

Corollary 4. Assume that $u \in C^{k+1+s}(\Omega)$ for $0 \leq s<\sigma$. With the assumptions of Theorem 2, the following estimate holds,

$$
\left|\nabla\left(u-u_{h}\right)(z)\right| \leq C h^{k}\left(\sum_{|\alpha|=k+1}\left|D^{\alpha} u(z)\right|+h^{s}\|u\|_{C^{k+1+s}(\Omega)}\right) .
$$




\section{Concluding Remarks}

We proved optimal $W_{\infty}^{1}$ error estimates for convex polyhedral domains in three dimensions. One of the main tools used in the proof are new Hölder type estimates for the Green's function on convex polyhedral domains. It is not difficult to see that if analogues Hölder estimates for the Green's function hold in higher dimensions then the same technique can be used to prove such optimal $W_{\infty}^{1}$ error estimates in higher dimensions.

The analysis carried out here can also be applied to discontinuous Galerkin (DG) methods. Using the local error estimates found in [5 and 14 and the techniques used here, we can prove optimal $W_{\infty}^{1}$ error estimates for various DG methods on convex polyhedral domains.

Recently Girault et al. [13] proved stability in $W_{\infty}^{1}$ norm for certain finite element methods for Stokes problem on polygonal and polyhedral domains. In three dimensions, $W_{p}^{2}$ regularity with $p>3$, was required for the velocity field. This leads to strong restrictions on the inner dihedral angles of the polyhedral domain, despite the fact that the derivatives of the velocity are Hölder continuous for general convex polyhedra (cf. 22 ). It would be interesting to see if the techniques used in this paper can be applied to Stokes problem in order to remove those restrictions. This is subject of ongoing work.

\section{REFERENCES}

[1] M. Asadzadeh, A.H. Schatz, and W. Wendland, Asymptotic error expansions for the finite element method for second order elliptic problems in $\mathbb{R}^{N}, N \geq 2$. I: Local interior expansions, preprint, Chalmers University of Technology, Göteborg University.

[2] M. Asadzadeh, A.H. Schatz, and W. Wendland, A non-standard approach to Richardson extrapolation in the finite element method for second order elliptic problems., preprint, Chalmers University of Technology, Göteborg University.

[3] S. Brenner and R. Scott, Mathematical Theory of Finite Element Methods, Springer-Verlag, New York, 1994.

[4] V. Carey, A posteriori error estimation via recovered gradients, Ph.D. Thesis, Cornell University, 2005.

[5] Z. Chen and H. Chen, Pointwise error estimates of discontinuous Galerkin methods with penalty for second-order elliptic problems, SIAM J. Numer. Anal. 42 (2004), 1146-1166.

[6] A. Demlow, Localized pointwise a posteriori error estimates for gradients of piecewise linear finite element approximations to second-order quasilinear elliptic problems, SIAM J. Numer. Anal. 44 (2006), no. 2, 494-514.

[7] A. Demlow, Weighted residual estimators for a posteriori estimation of pointwise gradient errors in quasilinear elliptic problems, preprint

[8] A. Demlow, Sharply localized pointwise and $W_{\infty}^{-1}$ estimates for finite element methods for quasilinear problems, Math. Comp. 76 (2007), 1725-1741.

[9] J. Douglas Jr. and T. Dupont, A Galerkin Methods for a Nonlinear Dirichlet Problem, Math. Comp. 29 (1975), 689-696.

[10] T. Dupont and R. Scott, Polynomial Approximation of Functions in Sobolev Spaces, Math. Comp. 34 (1980), 441-463.

[11] J. Frehse and R. Rannacher, Asymptotic $L^{\infty}$-error estimates for linear finite element approximations of quasilinear boundary value problems, SIAM J. Numer. Anal. 15 (1978), no. $2,418-431$.

[12] S. Fromm, Potential space estimates for Green potentials in convex domains, Proc. Amer. Math. Soc. 119 (1993), no. 1, 225-233.

[13] V. Girault, R.H. Nochetto, and R. Scott, Maximum-norm stability of the finite element Stokes projection, J. Math. Pures Appl. (9) 84 (2005), 279-330.

[14] J. Guzmán, Pointwise error estimates for discontinuous Galerkin methods with lifting operators for elliptic problems, Math. Comp. 75 (2006), 1067-1085. 
[15] M. Grüter and K.-O. Widman, The Green function for uniformly elliptic equations, Manuscripta Math., 37 (1982), 303-342.

[16] W. Hoffmann, A.H. Schatz, L.B. Wahlbin, and G. Wittum, Asymptotically exact a posteriori estimators for the pointwise gradient error on each element in irregular meshes. Part 1: A smooth problem and globally quasi-uniform meshes, Math. Comp. 70 (2001), 897-909.

[17] V.A. Kozlov, V.G. Maz'ya, and J. Rossmann, Spectral problems assosoated with corner singularities of solutions to elliptic equations, Mathematical Surveys and Monographs 85, Amer. Math. Soc., Providence, Rhode Island 2001.

[18] J.P. Krasovskii, Properties of Green's function and generalized solutions of elliptic boundary value problems, Soviet Mathematics (Translations of Doklady Academy of Sciences of the USSR) $10^{2}$ (1969), 54-120.

[19] V.G. Maz'ya and J. Roßmann, On the Agmon-Miranda maximum principle for solutions of elliptic equations in polyhedral and polygonal domains, Ann. Global Anal. Geom. 9 (1991) 3, 253-303.

[20] V.G. Maz'ya and J. Roßmann, Point estimates for Green's matrix to boundary value problems for second order systems in a polyhedral cone, Z. Angew. Math. Mech. 82 (2002) 5, 291-316.

[21] V.G. Maz'ya and J. Roßmann, Weighted Lp estimates of solutions to boundary value problems for second order elliptic systems in polyhedral domains, Z. Angew. Math. Mech., 83, no. 7, $435-467$.

[22] V.G. Maz'ya and J. Roßmann, Schauder estimates for solutions to a mixed boundary value problem for the Stokes system in polyhedral domains, Math. Methods Appl. Sci. 29 (2006), 965-1017.

[23] F. Natterer, "Uber die punktweise Konvergenz finiter Elemente, Numer. Math. 25 (1975), $67-77$.

[24] J.A. Nitsche, $L_{\infty}$ convergence of finite element approximations, Proceedings Second Conference on Finite Elements, Rennes, France (1975).

[25] J.A. Nitsche, $L_{\infty}$ convergence of finite element approximations, Mathematical Aspects of Finite Element Methods, Lecture Notes in Math., vol. 606, Springer-Verlag, 1977, 261-274.

[26] J.A. Nitsche and A.H. Schatz, Interior estimates for Ritz-Galerkin methods, Math. Comp. 28 (1974), 937-958.

[27] R. Rannacher, Zur $L^{\infty}$-Konvergenz linearer finiter Elemente beim Dirichlet-Problem, Math. Zeitschrift, 149 (1976), 69-77.

[28] R. Rannacher and R. Scott, Some Optimal Error Estimates for Piecewise Linear Finite Element Approximations, Math. Comp. 158 (1982), 437-445.

[29] P. Saavedra, R. Scott Variational formulation of a model free-boundary problem, Math. Comp. 57 (1991), 451-475.

[30] A. H. Schatz, A Weak Discrete Maximum Principle and Stability of the Finite Element Method in $L_{\infty}$ on the Plane Polygonal Domains. I, Math. Comp. 34 (1980), 77-91.

[31] A. H. Schatz, Pointwise error estimates and asymptotic error expansion inequalities for the finite element method on irregular grids: Part 1, Math. Comp. 67 (1998), 877-899.

[32] A. H. Schatz, Pointwise error estimates and asymptotic error expansion inequalities for the finite element method on irregular grids. II. Interior estimates, SIAM J. Numer. Anal. 38 (2000), no. 4, 1269-1293.

[33] A. H. Schatz, Perturbations of forms and error estimates for the finite element method at a point, with an application to improved superconvergence error estimates for subspaces that are symmetric with respect to a point, SIAM J. Numer. Anal. 42 (2005), no. 6, 2342-2365.

[34] A.H. Schatz and L.B. Wahlbin, Interior maximum norm estimates for finite element methods, Math. Comp. 31 (1977), 414-442.

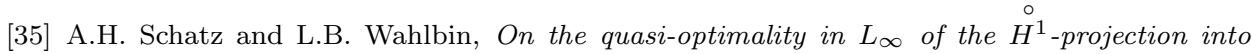
finite element spaces, Math. Comp. 38 (1982), 1-22.

[36] A.H. Schatz and L.B. Wahlbin, Interior maximum norm estimates for finite element methods II, Math. Comp. 64 (1995), 907-928.

[37] R. Scholz, A mixed method for 4th order problems using linear finite elements, RAIRO Anal. Numer. 12 (1978), no. 1, 85-90.

[38] L. R. Scott, Optimal $L_{\infty}$ estimates for the finite element method, Math. Comp. 30 (1976), 681-697. 
School of Mathematics, University of Minnesota, Minneapolis, MN 55455, USA.

E-mail address: guzma033@umn.edu

Department of Mathematics, University of Connecticut, Storrs, CT 06269, USA.

E-mail address: leykekhman@math.uconn.edu

Institut für Mathematik, Universität Rostock, 18051 Rostock, Germany.

E-mail address: juergen.rossmann@uni-rostock.de

Department of Mathematics, Cornell University, Ithaca, Ny 14853.

E-mail address: schatz@math.cornell.edu 